\title{
MRI chemical shift artifact produced by center-out radial sampling of k-space: a potential pitfall in clinical diagnosis
}

\author{
Mark Bydder ${ }^{1}$, Michael Carl $^{2}$, Graeme M. Bydder ${ }^{3,4}$, Jiang Du ${ }^{3}$ \\ ${ }^{1}$ Department of Radiological Sciences, David Geffen School of Medicine, University of California Los Angeles, CA, USA; ${ }^{2}$ Global MR Application \\ and Workflow, GE Healthcare, San Diego, CA, USA; ${ }^{3}$ Department of Radiology, University of California San Diego, San Diego, CA, USA; \\ ${ }^{4}$ Department of Radiology, The Christie NHS Foundation Trust, Manchester, UK
}

Correspondence to: Mark Bydder. Department of Radiological Sciences, David Geffen School of Medicine, UCLA, 924 Westwood Boulevard, Suite 615, Los Angeles, CA 90024, USA. Email: markbydder@gmail.com.

\begin{abstract}
Background: Center-out radial sampling of k-space in magnetic resonance imaging employs a different direction for each readout. Off-resonance artifacts (including those produced by chemical shift between water and fat) found with this type of sampling are usually described as blurring, however more specific characterization of these artifacts can be ascertained from the fact that their point spread function is ringshaped. This produces effects that differ from those seen with Cartesian sampling of k-space. Experiments were designed to demonstrate the origin of these artifacts and a volunteer was imaged to show them.

Methods: Two phantoms containing oil in a syringe and an annulus of oil surrounded by water were scanned with a range of bandwidths from 62.5 down to $4 \mathrm{kHz}$. In a human volunteer, head, pelvis and spine images were obtained with bandwidths of 62.5 and $4 \mathrm{kHz}$.

Results: The two phantoms showed displacement of the oil signal away from the center into the region of the surrounding water. The effect increased as the bandwidth was decreased. In the head of the volunteer, signal from fat in red bone marrow in the skull was displaced centrally and peripherally relative to water within the marrow, and appeared in the region between the skull and the brain, as well as in the surrounding scalp. Displacements of the former type simulated subdural hematomas. Displacement of perivesical fat signal centrally over the wall of the bladder simulated bladder tumor, and displacement of fat signal from red bone marrow in the lumbar spine to the intervertebral discs simulated their cartilaginous endplates.

Conclusions: Center-out radial artifacts are important to recognize on clinical images since they may mimic anatomy and simulate pathology. The article shows how these artifacts originate, includes examples, and describes how the artifacts differ from Cartesian chemical shift artifacts.
\end{abstract}

Keywords: Radial center-out sampling of k-space; fat imaging; chemical shift artifact; magnetic resonance imaging

Submitted Jan 28, 2021. Accepted for publication Mar 22, 2021.

doi: $10.21037 /$ qims-21-115

View this article at: http://dx.doi.org/10.21037/qims-21-115

\section{Introduction}

The chemical shift artifacts between water and fat that occur with conventional Cartesian sampling of k-space usually manifest as constant displacement of fat relative to water (1).
Since the magnitude and direction of the gradient used for frequency encoding with this type of sampling is identical for all phase encoding lines, chemical shift artifacts appear as displacement of fat signals relative to water signals in the

$\wedge$ ORCID: 0000-0001-9210-0225. 

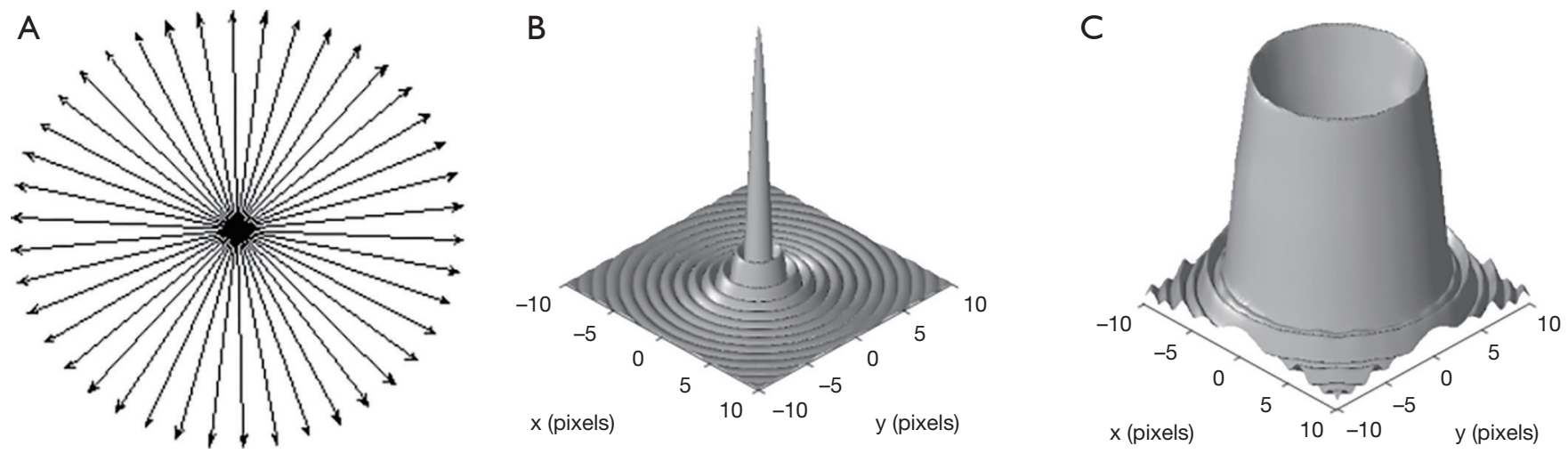

Figure 1 Schematic of (A) center-out radial trajectory for sampling k-space. The gradient directions are indicated by arrows. (B) shows the PSF for a low bandwidth acquisition on-resonance with this sampling. (C) shows the PSF for the same acquisition with the addition of offresonance effects from the chemical shift. (B) shows a sharp central peak which is surrounded by circular rings (Gibbs ringing). (C) shows no central peak and complete displacement of signal away from the center, as well as larger surrounding rings.

frequency encoding direction. With radial and other types of non-Cartesian sampling of k-space, where the direction of the frequency encoding changes, the appearance of chemical shift artifacts between fat and water is less specific and not so well known. This is a potential problem since non-Cartesian techniques are being used more frequently in clinical practice (2). Ultrashort echo time (UTE) imaging is a particular example of non-Cartesian imaging which is used in musculoskeletal, lung and neurological imaging $(3,4)$. It employs center-out sampling of $\mathrm{k}$-space which is a type of radial sampling (Figure 1A).

The concept of the point spread function (PSF) is useful in imaging systems for illustrating the imperfections in the encoding and/or reconstruction of such systems. Essentially the PSF is the image that would be obtained by encoding and reconstructing an infinitely narrow point object. Ideally, the result should be a single bright pixel, however practical limitations mean that the image and the object are always different. In particular, the finite resolution of the measuring apparatus causes the infinitely narrow object to appear with a finite width (i.e., the point is spread out on the image). In this situation, the width of the object on the image is a property of the measuring device.

Other artifacts, such as chemical shift, aliasing and blurring, can also be represented in terms of a PSF, e.g., in Cartesian imaging the PSF for chemical shift is a displacement of the point from its true location. The PSF can be calculated numerically given a physical model of the system. In the case of center-out radial sampling, PSFs are shown in Figure 1B,C for the case of an on-resonance and an off-resonance point, respectively. These illustrate both the finite resolution of the scanner (Figure 1B) and a chemical shift effect added to this (Figure 1C).

UTE imaging is often used to show signal from short (1-10 ms) and ultrashort $(0.1-1 \mathrm{~ms}) \mathrm{T}_{2}$ water containing tissues such as cortical bone, periosteum, entheses, the calcified and deep radial layers of articular cartilage, as well as cartilaginous EndPlates (CEPs) of intervertebral discs. Ultrashort $\mathrm{T}_{2}$ tissues or structures do not show signal with conventional longer TE sampling techniques since the signal has decayed to zero before data is collected (5). Chemical shift artifacts arising from fat may be displaced into regions of zero signal on long TE images and be mistaken for real tissues when UTE imaging is used. This is particularly likely when ultrashort $T_{2}$ tissues are shown after UTE imaging is first implemented and signal is seen. It may not be obvious whether this signal is a normal appearance or an artifact. Many short and ultrashort $\mathrm{T}_{2}$ tissues are adjacent to fat, and image interpretation at these boundaries may be particularly problematic.

Image blurring, horseshoe and other artifacts have been described with radial sampling of k-space (1-8) but this does not describe the full range of artifacts that may be seen with this type of sampling. The off-resonance effect in center-out radial sampling of $\mathrm{k}$-space creates a ringshaped PSF (Figure 1C) $(9,10)$. While this is widely known, there is a need to show how this manifests as chemical shift artifacts on images, and whether or not these artifacts can mimic normal structures and/or disease in clinical practice. The purpose of this paper is to unequivocally demonstrate 

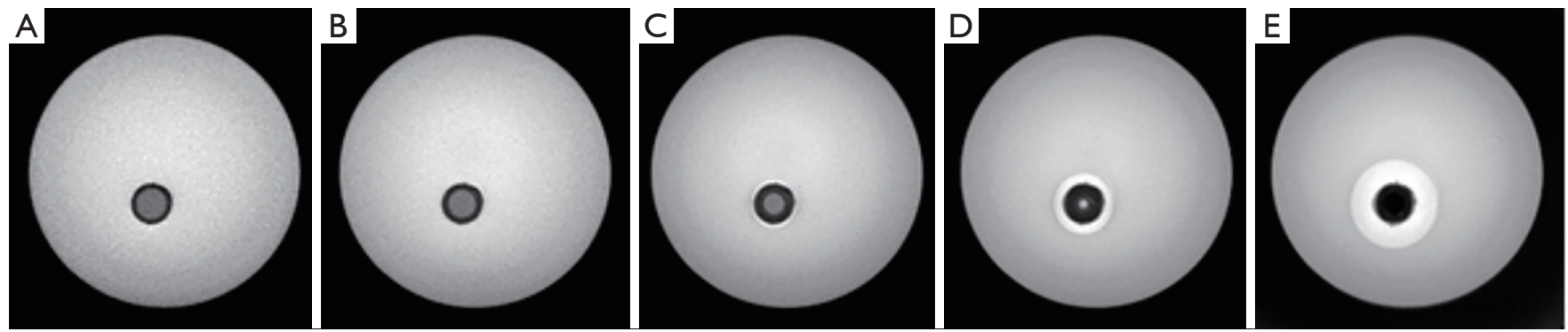

Figure 2 Central oil sample in a syringe surrounded by water. Matrix 256 $6^{2}$ FOV $15 \mathrm{~cm}$, TE $2.3 \mathrm{~ms}$, bandwidth 62.5 (A), 31 (B), 15 (C), 8 (D) and 4 (E) kHz. (A) shows radially symmetric signal from the off-resonance oil which is mid-gray. It is surrounded by a dark ring where the syringe produces no detectable signal. As the bandwidth is decreased (B) to (D) less central mid-gray signal is seen from the oil and the water surrounding it increases in signal as signal from the oil is displaced outwards and added to it. At $4 \mathrm{kHz}$ (E) no signal from the oil in the syringe remains.

the artifacts specifically due to radial center-out sampling of k-space with UTE imaging in phantoms, show their appearance in normal human images and assess their potential for misdiagnosis.

\section{Methods}

Data were acquired on a Twinspeed $3 \mathrm{~T}$ scanner (GE Healthcare, WI) using a 2D UTE sequence with center-out radial sampling. Image reconstruction was performed online using standard Kaiser-Bessel regridding. Slice selective sinc excitation was used with a TE of $2.3 \mathrm{~ms}$ to render fat and water signals in-phase, or close to in-phase at $3 \mathrm{~T}$. A flip angle of 90 degrees and TR $=1,000 \mathrm{~ms}$ were used. A range of bandwidths from 62.5 to $4 \mathrm{kHz}$ was used to manipulate the appearance of the chemical shift artifact over the imaged field of view.

\section{Phantom studies}

Two circular phantoms were constructed from vegetable oil (to simulate in vivo triglyceride) and water doped with Gadobenetate dimeglumine (Multihance, Bracco Diagnostics, NJ). The first was made with a syringe containing oil placed inside a water filled beaker to show effects from a "point" source of signal. The second phantom was made using three beakers of different sizes in a "Russian doll" arrangement, with the largest beaker containing the medium-sized beaker and this containing the smallest beaker. The largest and smallest beakers contained water, and the middle-sized beaker contained vegetable oil. The purpose of this phantom was to show artifacts arising from structures comparable to those seen in vivo with a layer of fat surrounded on either side by water (as in urine), and/or water containing tissues.

\section{Human volunteer}

A human volunteer (male, age 65 years) was scanned using the same imaging sequence and range of bandwidths as for the phantoms. Representative examples of images of the head, pelvis and spine were chosen. Imaging was performed with Institutional Review Board approval from the University of California, San Diego and written informed consent.

\section{Results}

Figure 2 shows results from the first phantom containing oil centrally within a syringe. This reveals radially symmetric signal from the off-resonance oil which is mid-gray in (Figure 2A). It is surrounded by a dark ring where the syringe produces no detectable signal. As the bandwidth is decreased (Figure 2B-D) less central mid-gray signal is seen and the water surrounding it increases in signal as the signal from the oil is progressively displaced outwards into it. At $4 \mathrm{kHz}$ (Figure 2E) no signal from the oil remains in the syringe at the correct location of the oil.

Figure 3 shows results from the second phantom which had an oil annulus surrounded by water in the two beakers internal and external to it. In Figure $3 A$ there is a central ring of signal from the oil which appears mid-gray. It is surrounded by narrow inner and outer dark rings from the material of the beakers. As the bandwidth decreases (Figure 3B-E) the central mid-gray ring becomes thinner 

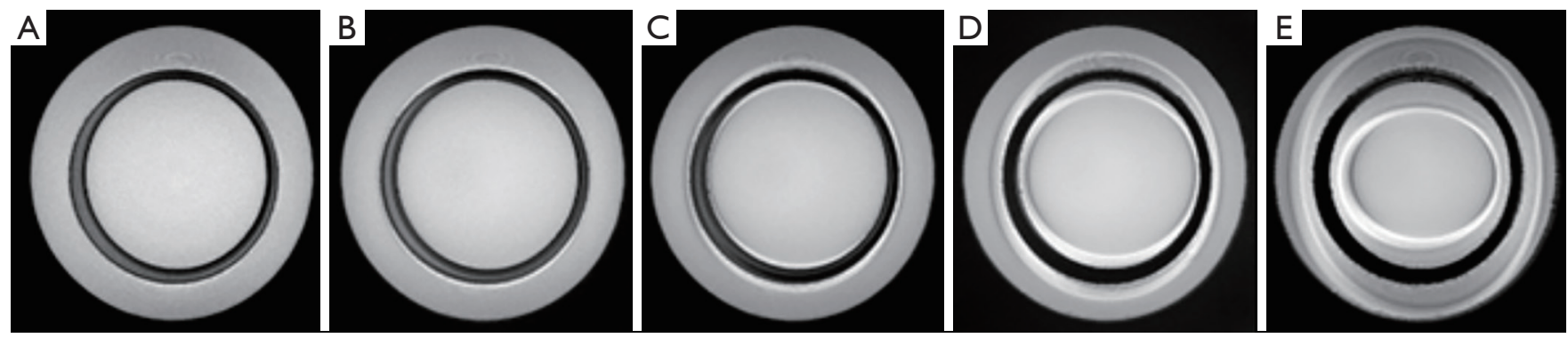

Figure 3 Oil annulus situated between outer and inner beakers containing water. Matrix 256 $6^{2}$ FOV $15 \mathrm{~cm}$, TE 2.3 ms, bandwidth 62.5 (A), 31 (B), 15 (C), 8 (D) and 4 (E) kHz. In (A) there is a central ring containing oil which appears mid-gray. It is surrounded by inner and outer dark rings from the material of the two surrounding beakers. As the bandwidth is progressively decreased (B-E) the central mid-gray oil ring becomes thinner and less obvious, until it is no longer seen in (E). There is an increase in signal in the surrounding internal and external water which becomes more extensive from (B) to (E). There are slight asymmetries to the artifact which are most evident at the lowest bandwidth, and reflect susceptibility-induced off-resonance effects in the regions of the interfaces between the oil and water layers.

and less obvious, until it is no longer seen in (Figure 3E). There is an increase in signal in the surrounding internal and external water which becomes more extensive from Figure $3 B-E$. There are slight asymmetries to the artifact which are most evident at the lowest bandwidth and reflect susceptibility-induced off-resonance effects at the interfaces between the oil and water layers.

Figure $4 A$ is a transverse image of the head of the volunteer with $62.5 \mathrm{kHz}$ bandwidth and Figure $4 B$ is the same slice imaged at $4 \mathrm{kHz}$. The latter Figure shows the brain with areas of increased signal adjacent to it inside the skull. These areas (arrows) simulate the appearance of subdural hematomas. In addition, in Figure $4 B$ an increased number of layers is seen in the surrounding scalp. Signal from fat in the red bone marrow (approximately 50\%) between the inner and outer tables of the skull is displaced centrally inside the skull around the brain, and peripherally outside the skull into the scalp. Unlike Cartesian chemical shift artifacts which are seen in one direction only and have a single displacement polarity, the radial center-out artifacts are seen in multiple directions and have two displacement polarities (i.e., both central and peripheral displacement of signal).

Figure $5 A$ shows a transverse image of the pelvis of the volunteer at $62.5 \mathrm{kHz}$. Figure $5 B$ shows the same slice at $4 \mathrm{kHz}$ bandwidth. In this image, additional high signal is seen between the urine in the bladder and the surrounding pelvic tissues (arrows) giving the appearance of bladder wall thickening. This simulates bladder tumor. The artifacts are seen in different directions and result from displacement centrally of signal from more peripheral perivesical fat.
Figure $6 A$ is a sagittal image of the lumbar spine of the volunteer obtained with a bandwidth of $62.5 \mathrm{kHz}$. Figure $6 B$ is the same slice imaged with a bandwidth of $4 \mathrm{kHz}$. In the latter image high signal is seen at the upper and lower margins of the intervertebral discs (arrows) between the vertebral bodies. The appearances simulate those of CEPs seen with UTE subtraction imaging. The CEP has a zero signal with conventional long TE imaging, but shows low signal with UTE imaging. When a short TE zero signal image is subtracted from the low signal UTE image, relatively higher signal is seen in the CEPs. This genuine finding is simulated by the chemical shift artifacts shown in Figure 6B. Unlike Cartesian chemical shift artifacts using sagittal frequency encoding where increased signal is only seen in the region of one CEP, with radial center-out sampling increased signal is seen in the regions of both the upper and lower CEPs of intervertebral discs.

\section{Discussion}

Chemical shift between water and fat with center-out radial $\mathrm{k}$-space sampling can result in partial or complete loss of signal from the original site, as well as increases in central and peripheral signal around the original site of the fatty tissue. This may produce a reduction in the apparent size of the fatty tissue, and additional signals that extend beyond the original fatty tissue. At higher bandwidths the effect may appear like blurring, but at lower bandwidths the artifact can take the form of discrete well defined signals displaced away from the original site of the fat. These may simulate normal anatomy and/or disease. Similar effects 

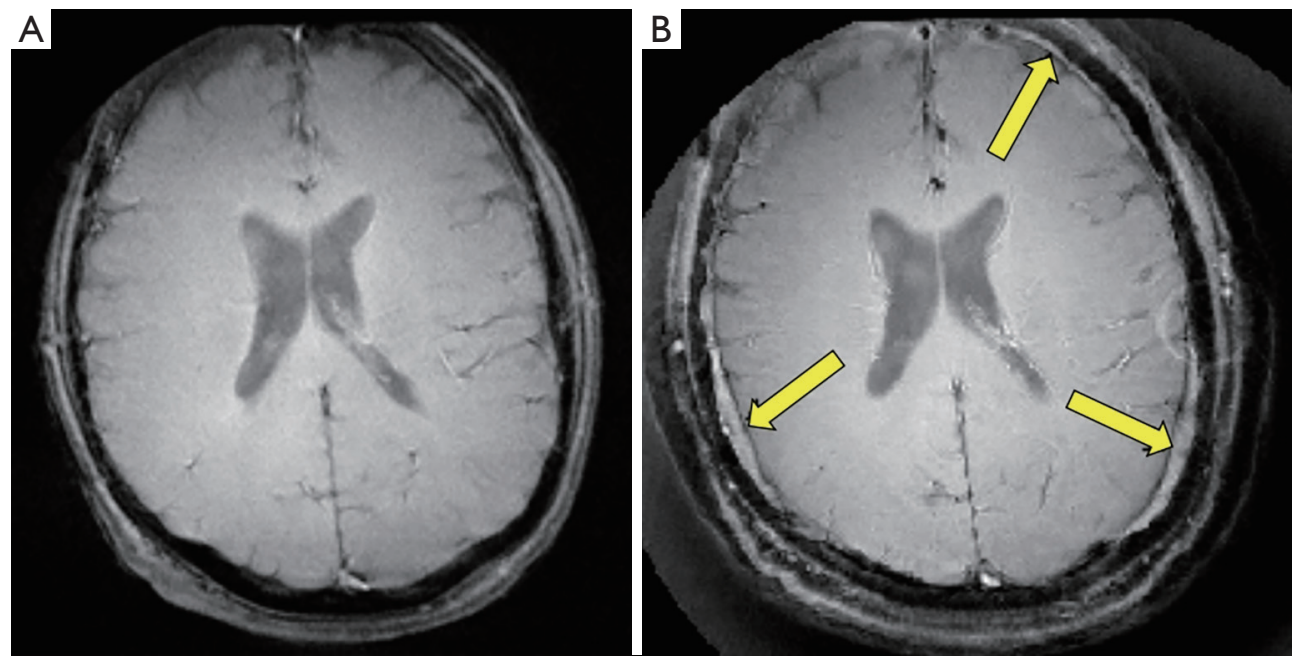

Figure 4 Images of the head of the volunteer. (A) A transverse image with $62.5 \mathrm{kHz}$ bandwidth and (B) the same slice imaged at $4 \mathrm{kHz}$ bandwidth. (B) The brain with areas of increased signal adjacent to it inside the skull (arrows). These areas simulate the appearance of subdural hematomas. In addition, an increased number of layers is seen in the surrounding scalp in (B). Signal from fat (approximately $50 \%$ ) in the red bone marrow between the inner and outer tables of the skull is displaced centrally inside the skull around the brain, and peripherally outside the scalp. Unlike Cartesian chemical shift artifacts which are seen in a single frequency encoding direction and have a single polarity, radial center-out artifacts are seen in different directions and have positive and negative polarities (i.e., central and peripheral displacement of signal).
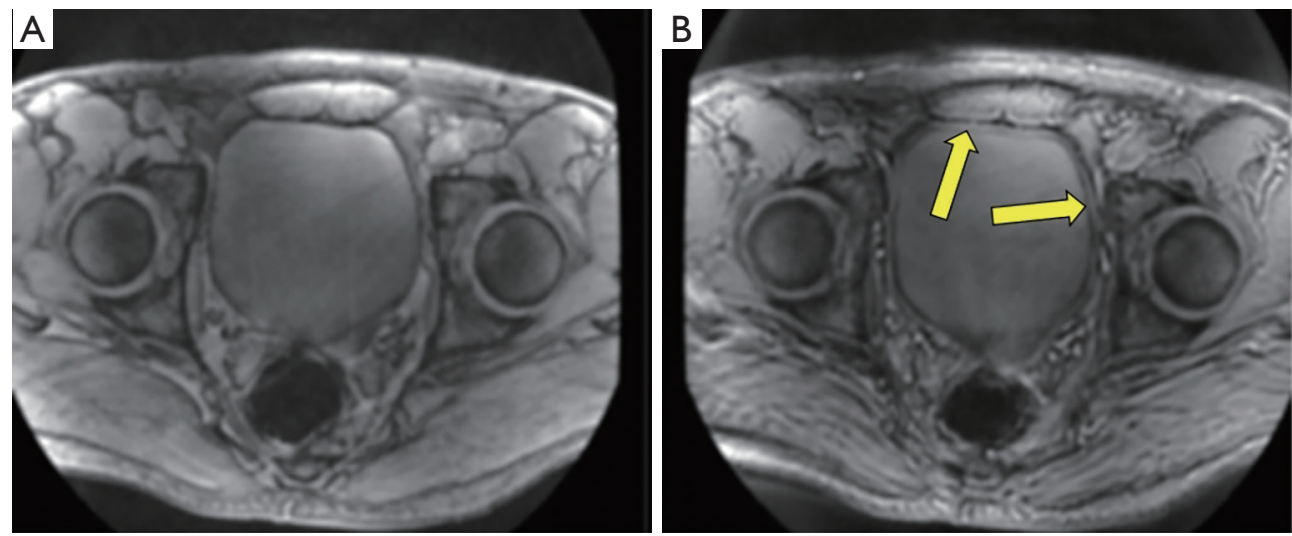

Figure 5 Images of the pelvis of the volunteer. (A) is a transverse image at $62.5 \mathrm{kHz}$; (B) shows the same slice at $4 \mathrm{kHz}$ bandwidth. Additional high signal is seen between the urine in the bladder and the pelvic tissues surrounding the bladder (arrows) giving the appearance of bladder wall thickening. This simulates changes due to tumor. The artifacts are seen in different radial directions and result from displacement centrally of signal from more peripheral perivesical fat.

are observed with off-resonance generally and may be used to enhance contrast (1). As expected from conventional Cartesian scanning, the artifact is more obvious at low receiver bandwidths and at high field strengths.

At external boundaries of the skeleton, center-out radial artifacts can produce a high signal intensity appearances which simulate periosteum or other tissues as well as a low signal regions that simulate subchondral bone. The mixed composition of red bone marrow with approximately $50 \%$ fat and $50 \%$ water means that the fat signal may be selectively displaced and produce artifacts that differ from those seen at boundaries between pure fat and pure water. 

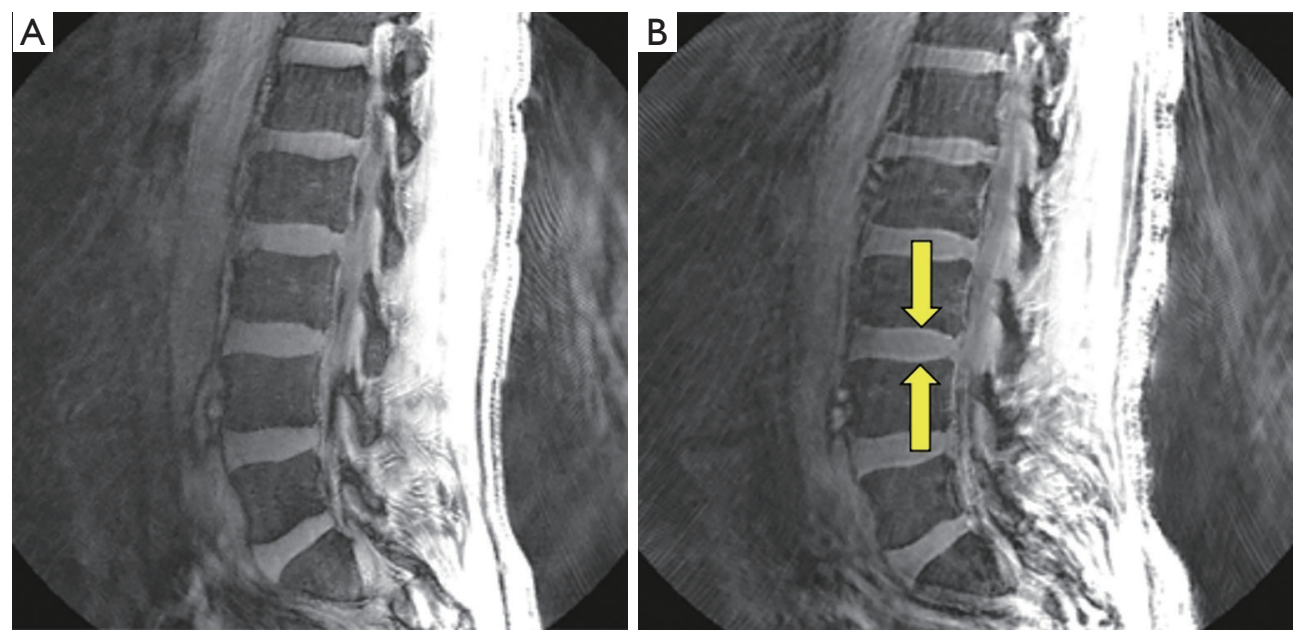

Figure 6 Images of the lumbar spine of the volunteer. (A) is a sagittal image obtained with a bandwidth of $62.5 \mathrm{kHz}$. (B) is the same slice imaged with a bandwidth of $4 \mathrm{kHz}$. In (B) high signal is seen at the upper and lower margins of the intervertebral discs adjacent to vertebral bodies above and below them (arrows). The appearances simulate the appearances of CEPs seen on UTE subtraction images. The CEP has a zero signal with conventional long TE imaging, but shows detectable relatively low signal with UTE imaging. When a longer TE image is subtracted from a UTE image relatively high signal results in the CEP. This genuine finding is simulated by the chemical shift artifacts shown in (B). Unlike Cartesian chemical shift artifacts using sagittal frequency encoding where increased signal is only seen in the region of one CEP, with radial center-out sampling increased signal is seen in both the upper and lower CEPs.

After experience with Cartesian chemical shift artifacts, it may be counterintuitive that the fat signal with radial center-out imaging is displaced both peripherally and centrally. In addition, the artifact is generally distributed radially, but it may appear as displacement mainly in a single direction when there is a linear water fat boundary as with the appearance simulating CEPs.

Fat saturation and inversion pulses with selective fat signal nulling can be used to suppress the fat signal to try and avoid chemical shift artifacts of this type but susceptibility effects at boundaries such as those between cortical bone, periosteum and surrounding soft tissues may render the fat suppression ineffective. In a study of the periosteum, Kim et al. found when using 3D UTE imaging that the high signal of normal periosteum overlapped that of artifacts both when fat suppression was not used, and when it was used (11).

If UTE center-out radial images are subtracted from longer TE Cartesian images, the resultant subtraction image may show chemical shift artifacts from both types of k-space sampling. If radial images at different TEs are acquired with different bandwidths, additional chemical shift artifacts may arise.

The TE of 2.3 ms was chosen so oil and water signals were in-phase (at $3.0 \mathrm{~T}$ ) and the signals would combine to give high pixel signal values. At TEs other than in-phase the oil and water signals exhibit interference phenomena and may combine destructively giving dark low pixel signal values. The in-phase case is similar to using an ultrashort TE close to zero. The reason for not using the shortest TE possible in these experiments was because of additional complications relating to UTE sequences that are found at very short TEs, namely, through-slice chemical shifts when using positive and negative half-excitations, variable rate slice-selection and short time-constant eddy currents (2) which may also produce artifacts. The initial purpose of this study was to specifically characterize and illustrate in-plane chemical shift artifacts that were unequivocally attributable to center-out radial sampling of $\mathrm{k}$-space. The examples illustrated were with signals from fat and water in phase, but if they were out of phase and the fat was displaced into areas of water signal this could lead to artifactual cancellation of signal and so confuse the analysis and demonstration of the center-out radial artifacts.

\section{Conclusions}

This paper provides a basis for understanding chemical shift artifact in center-out radial UTE imaging and demonstrates that these artifacts can simulate normal structures and disease. 


\section{Acknowledgments}

Funding: This study was supported by NIH (R01 NS092650, R01AR075825 and R01 AR062581), and GE Healthcare.

\section{Footnote}

Conflicts of Interest: All authors have completed the ICMJE uniform disclosure form (available at http://dx.doi. org/10.21037/qims-21-115). Dr. JD serves as an unpaid editorial board member of Quantitative Imaging in Medicine and Surgery. The other authors have no conflicts of interest to declare.

Ethical Statement: The authors are accountable for all aspects of the work in ensuring that questions related to the accuracy or integrity of any part of the work are appropriately investigated and resolved. The study was conducted in accordance with the Declaration of Helsinki (as revised in 2013). The study was approved by the Institutional Review Board of the University of California, San Diego and informed consent was obtained from the volunteer.

Open Access Statement: This is an Open Access article distributed in accordance with the Creative Commons Attribution-NonCommercial-NoDerivs 4.0 International License (CC BY-NC-ND 4.0), which permits the noncommercial replication and distribution of the article with the strict proviso that no changes or edits are made and the original work is properly cited (including links to both the formal publication through the relevant DOI and the license). See: https://creativecommons.org/licenses/by-nc-nd/4.0/.

\section{References}

1. Hood MN, Ho VB, Smirniotopoulos JG, Szumowski J.

Cite this article as: Bydder M, Carl M, Bydder GM, Du J. MRI chemical shift artifact produced by center-out radial sampling of k-space: a potential pitfall in clinical diagnosis. Quant Imaging Med Surg 2021;11(8):3677-3683. doi: 10.21037/ qims-21-115
Chemical Shift: The artifact and clinical tool revisited. Radiographics 1999;19:357-71.

2. Weiger M, Pruessman KP. Short-T2 MRI: Principles and recent advances. Prog Nucl Magn Reson Spectrosc 2019;114-115:237-270.

3. Bergin CJ, Pauly JM, Macovski A. Lung parenchyma: projection reconstruction MR imaging. Radiology 1991;179:777-81.

4. Robson MD, Gatehouse PD, Bydder M, Bydder GM. Magnetic resonance: an introduction to ultrashort TE imaging. J Comput Assist Tomogr 2003;27:825-46.

5. Mentrup D, Eggers H. Signal decay correction in 2D ultra-short echo time imaging. MAGMA 2006;19:62-70.

6. Rahmer J, Bornert P, Bos C. Three-dimensional radial ultrashort echo-time imaging with T2 adapted sampling. Magn Reson Med 2006;55:1075-82.

7. Altbach MI, Trouard TP, Van de Walle R, Theilmann RJ, Clarkson E, Barret HH, Gmitro AF. Chemicalshift imaging utilizing the positional shifts along the readout gradient direction. IEEE Trans Med Imaging 2001;20:1156-66.

8. Seevinck PR, de Leeuw H, Box C, Bakker CJG. Highly localized positive contrast of small paramagnetic objects using $3 \mathrm{D}$ center-out radial sampling with off-resonance reception. Magn Reson Med 2011;65:146-56.

9. Man LC, Pauly JM, Macovski A. Multi-frequency interpolation for fast off-resonance correction. Magn Reson Med 1997;37:785-792.

10. Haake EM, Brown RW, Thompson MR, Venkatesan R. Magnetic Resonance Imaging: physical principles and sequence design. New York: John Wiley \& Sons, 1999:327-328.

11. Kim DJ, Hwang K, Kim H, Cha JG, Jang H, Park JY, Kim YJ. Depiction of the periosteum using ultrashort echo time pulse sequence with three-dimensional cone trajectory and histologic correlation in a porcine model. Korean J Radiol 2021;22:782-91. 